\title{
Sistemas de Información Geográfica y su Análisis Aplicado en Zonas de Delincuencia en la Ciudad de México
}

\author{
Abraham Espinoza-Ramírez, Mariko Nakano, Gabriel Sánchez-Pérez, Antonio Arista-Jalife* \\ Instituto Politécnico Nacional, ESIME Culhuacán, Av. Santa Ana 1000, Coyoacán, CP. 04430 \\ Sección de Estudios de Posgrado e Investigación, Ciudad de México. \\ (e-mail: abraham_er@icloud.com,mnakano@ipn.mx, arista.antonio@gmail.com) \\ D \\ ${ }^{*}$ Autor a quien debe dirigirse la correspondencia
}

Recibido Feb. 13, 2018; Aceptado Abr. 26, 2018; Versión final May. 16, 2018, Publicado Oct. 2018

\section{Resumen}

Se propone un sistema computacional basado en dispositivos móviles que permite crear indicadores de delincuencia dentro de un plano cartográfico. Con este sistema, el usuario final será capaz de identificar, asimilar y evitar el delito, así como para colaborar con su disminución y erradicación. El trabajo se desarrolla en el marco del notorio aumento en la percepción de inseguridad por parte de los ciudadanos de ciudad de México, de acuerdo al informe de índice de paz México 2017. Como resultado del estudio, se proveen modelos de mapas con incidentes geo-referenciados, por medio de un sistema de información geográfico como herramienta principal. También se hace uso de la geolocalización y posicionamiento móvil, con el propósito de contribuir al monitoreo de la delincuencia, detectar los percances de forma temprana, y servir como base para la toma de decisiones en materia de seguridad.

Palabras clave: mapeo del crimen; sistema de información geográfica SIG; delincuencia; crimen en México; crowdsourcing.

\section{Geographic Information Systems and their Analysis Applied in Crime Areas in Mexico City}

\begin{abstract}
A computational system based on mobile devices that allows to create delinquency indicators in a cartographic map is proposed. With this system, the end user will be capable of identifying, assimilating and avoiding delinquency, as well as collaborating with its reduction and eradication. The work is motivated by the notorious increment of the insecurity perception of the citizens of Mexico City, as stated on the Mexico peace index report of 2017. As a result of the study, models of maps with geo-referenced incidents are provided. Also, it makes use of geo-location and mobile positioning, with the objective of contributing to the surveillance of delinquency, detecting earlier the undesirable events and serve as basis for decision making in terms of security.
\end{abstract}

Keywords: crime mapping; geographic information systems GIS; delinquency; crime in México; crowdsourcing. 


\section{INTRODUCCIÓN}

En la actualidad, México dedica grandes esfuerzos en contención de violencia -de acuerdo al informe de índice de la paz México 2017- colocándolo dentro de los diez primeros países que mayores recursos destina a este rubro en la región de Latinoamérica. El costo por contención de la violencia en el país se debe a diversos factores como la desconfianza en las fuerzas policiales y judiciales (Cruz, 2012), lo que eleva la percepción de inseguridad y causa que los ciudadanos teman el denunciar y dar parte a las autoridades. Las tasas delictivas en la Ciudad de México son elevadas y los retos a resolver numerosos. El modus operandi más común es el robo en medios de transporte (Vilalta, 2011) y asalto al transeúnte, mismo que continúa en crecimiento; aunado a ello, no se cuentan con datos cartográficos precisos que permitan generar soluciones preventivas y proactivas en diversos puntos de la Ciudad de México. Como muestra, en la figura 1 se observa un análisis de la modificación de percepción pública de la paz en México desde al año 2003 al año 2016, creado por el informe de índice de la paz México 2017.

La Ciudad de México es un área proclive para la delincuencia organizada y no organizada debido a la conglomeración económica que presenta, al igual que su capacidad mercantil cambiante y dinámica, lo que causa diversas actividades ilícitas que permiten la generación de negocios criminales de escala regional, nacional e inclusive internacional. (Alvarado, 2016). Uno de los retos importantes para México es la reducción de los niveles de impunidad: Grupos delictivos operan debido a las actividades ilícitas que ocurren en barrios de la Ciudad de México que encuentran facilidad de generarse debido al clima de protección, extorsión y robo impune. Aunado a ello, la actividad delictiva genera ganancias atractivas (Alvarado, 2016) que causan la proliferación y expansión en poblaciones vulnerables económicamente.

Un análisis presentado en el trabajo de (Bailey, 2014) arrojan que la inseguridad en México es un flagelo que no solamente estanca el progreso del país, sino que es una de las causas fundamentales de una democracia de calidad deficiente, una percepción más intensa en el incumplimiento de la ley, y un comportamiento destructivo del crimen, exacerbado por la corrupción y la violencia. En la entrevista con John Bailey (Ruiz, 2015) se menciona un tipo de criminalidad peligrosa: La criminalidad territorial, misma que al llegar causan extorsiones, controlan a las fuerzas policiales, corrompen y penetran los sistemas policiales y judiciales. Por ende, son más peligrosos ya que sus alcances no pueden ser adecuadamente delimitados: Son capaces de corromper instituciones públicas y privadas de igual manera.

Un mecanismo que ha implementado la autoridad para hacerle frente a la problemática referente a la seguridad es crear mecanismos que faciliten una denuncia anónima, como la implementación de un número telefónico universal para emergencias y tecnología móvil para realizar denuncias, como es la aplicación móvil 9-1-1 implementada por el gobierno mexicano. Sin embargo este trabajo plantea la hipótesis de que los esfuerzos en materia de prevención del crimen podrían potencializarse por medio del uso de la tecnología basada en fuentes sociales, también conocida como crowdsourcing: actividades colaborativas basadas en la comunicación de internet, que permiten la creación e innovación basada en el usuario (Estellés-Arolas, 2012)

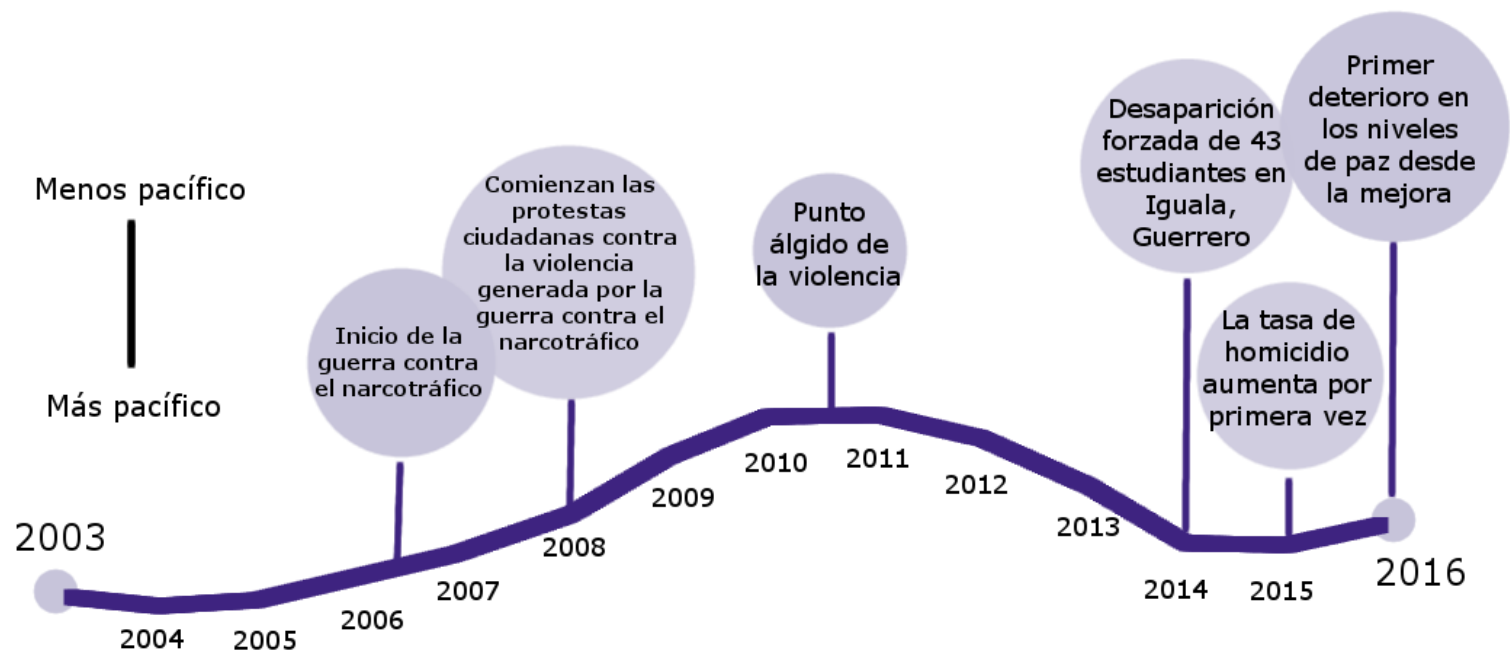

Fig.1. Gráfica de tendencia de la paz en México, generada de 2003 a 2016

Este tipo de interacción colaborativa provee lo que los pioneros del tema le llaman la sabiduría del grupo: un tipo de inteligencia colectiva que puede agregar información y resolver problemas por medio de la colaboración de cada uno de los usuarios. El beneficio no solamente recae sobre el sistema, sino sobre las mismas personas que la utilizan, siendo ellos mismos los primeros beneficiados en su implementación. 
(Brabham, 2008). Esto conlleva a que a mayor cantidad de usuarios empleando del sistema, mayor inteligencia y utilidad emana de este.

Este tipo de esquemas colaborativos ha sido utilizado con antelación para resolver distintas problemáticas, como es el caso de la coordinación y respuesta ante desastres naturales (Gao, 2011). La búsqueda e investigación de nuevos productos farmacéuticos (Ekins, 2010), e inclusive en efectivos métodos de reingeniería de procesos orientados a negocios (Khasraghi, 2012). En aras de hacer frente a la criminalidad territorial, la cual es su variante más peligrosa, este artículo presenta un sistema computacional que tiene como funcionalidad principal el generar una cronología de eventos con su respectiva referencia geográfica, basado en el modelo de crowdsourcing y la popular plataforma de desarrollo Android OS. Diversos objetivos sirven como motivación para la creación de dicho sistema, entre ellos la recolección de datos para mostrar al usuario final los espacios públicos más seguros, permitiendo el compartir a través de un mapa los reportes delictivos cercanos de otros usuarios.

Como se ha mencionado, el sistema descrito dentro de este artículo tiene la finalidad de basar su potencialidad en el esquema de crowdsourcing: el resultado de su utilización son las estadísticas delictivas y el sistema de alerta comunitaria, lo que permite combatir a la delincuencia para evitar situaciones de riesgo, al igual que la ayuda mutua entre autoridades y ciudadanos para la denuncia y erradicación del delito. Otros de los objetivos de este sistema es el análisis de la delincuencia en planos cartográficos por medio de la colaboración de los usuarios, lo que no solamente permitiría a los ciudadanos el evadir zonas con alta densidad criminalística, sino a las autoridades el implementar medidas de prevención y seguridad por medio del análisis del crimen, definido como el proceso de utilizar un sistema de información geográfica (SIG) en combinación con técnicas de análisis para centrarse en el contexto espacial de las actividades penales y otras actividades de aplicación de la ley (Boba, 2001).

Este sistema puede ser utilizado no solamente en la Ciudad de México -el cual es el caso de estudio de este trabajo- sino en cualquier ciudad que requiera esfuerzos en materia de seguridad. Por ende el propósito de este trabajo consiste en la captura tanto espacial (latitud / longitud) como temporal (fecha y hora, o bien, tiempo real) del delito ocurrido, de tal manera que estos esfuerzos generen una base de datos y un mapa cartográfico que permita viajes más seguros en la megalópolis donde este sistema sea implementado. Para acotar el alcance del modelo presentado, se implementará su utilización en la demarcación de la alcaldía de Iztacalco, dentro de la Ciudad de México.

Modelos similares se han llevado a cabo para estudiar la seguridad pública en las grandes ciudades como Xianmen, China (Yan, 2015) en donde se utilizan sistemas distintos, como la extracción de datos por diarios electrónicos. En otros trabajos se ha abordado el uso de tecnologías móviles para que los usuarios puedan alertar a las fuerzas policiales (Zhong, 2012). O bien, su uso para la comunicación y la asistencia del trabajo de campo de dichas fuerzas (Duansheng, 2009). Otros trabajos emplean técnicas de minería de datos para extraer información relevante de reportes policiales y colocarlos dentro de sistemas de información geográfica (Fan, 2009). Sin embargo, no se ha abordado una estrategia de crowdsourcing con sistemas móviles para dotar de diferentes fuentes de información a los sistemas de seguridad.

El presente trabajo está organizado de la siguiente forma: En la sección Sistema frontal del usuario, se describe el proceso de extracción, clasificación y empaquetado de los datos obtenidos por los usuarios dentro de la aplicación. En la sección subsecuente, titulada Metodología: Tratamiento de la información se mencionaran las fases de recolección de datos, almacenamiento y utilización para determinar acciones preventivas. Como tercera sección, se presentan los resultados en forma de un mapa temático que permite la interpretación y visualización de los datos, mientras que en la cuarta sección se realiza una discusión final del trabajo y posibles alcances futuros. Finalmente, en las conclusiones, se presentan los objetivos alcanzados.

\section{SISTEMA FRONTAL DEL USUARIO}

Los sistemas en dispositivos móviles tienen el objetivo de emplear los recursos embebidos en la plataforma para asistir al usuario final. La aplicación propuesta -GeoSecurityMX- no es la excepción, ya que para alcanzar la finalidad de asistir en tiempo real al usuario, emplea la tecnología de geo-localización por el sistema de posicionamiento global (GPS), así como las tecnologías de comunicación inalámbrica (Wi-Fi o bien una red de telecomunicaciones $4 \mathrm{G})$.

El usuario al descargar la aplicación y registrar sus datos en ella, es capaz de elegir el tipo de crimen a reportar: robo a persona, vehículo, casa/habitación, u homicidio, además de una descripción detallada del suceso, y la información temporal (fecha y hora) aproximada del percance, como se muestra en la figura 2. 
Una vez obtenidos los datos mencionados, el sistema propuesto recolecta la información del dispositivo, con la finalidad de obtener la ubicación en tiempo real del usuario en el momento del reporte.

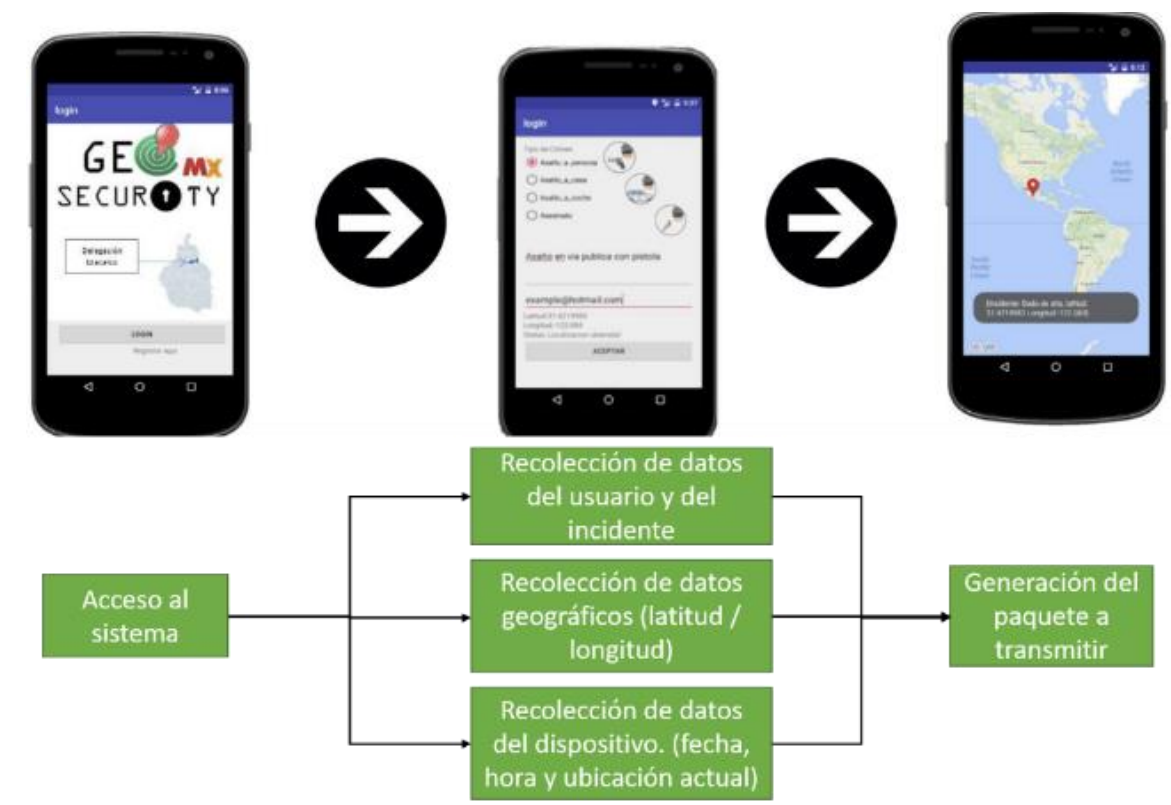

Fig. 2. Algoritmo de recolección de datos y generación del paquete de información.

Una vez adquiridos los datos del usuario, los datos del percance, y la geo-localización del dispositivo, esta información es enviada a un servidor de recolección por medio del protocolo TCP/IP, lo que permite utilizar cualquiera de las redes de telecomunicaciones (ya sea Wi-Fi o 4G), el paso descrito se muestra en la figura 3. Dentro del servidor, los datos son procesados según la estrategia de tratamiento de la información descrita en la siguiente sección.

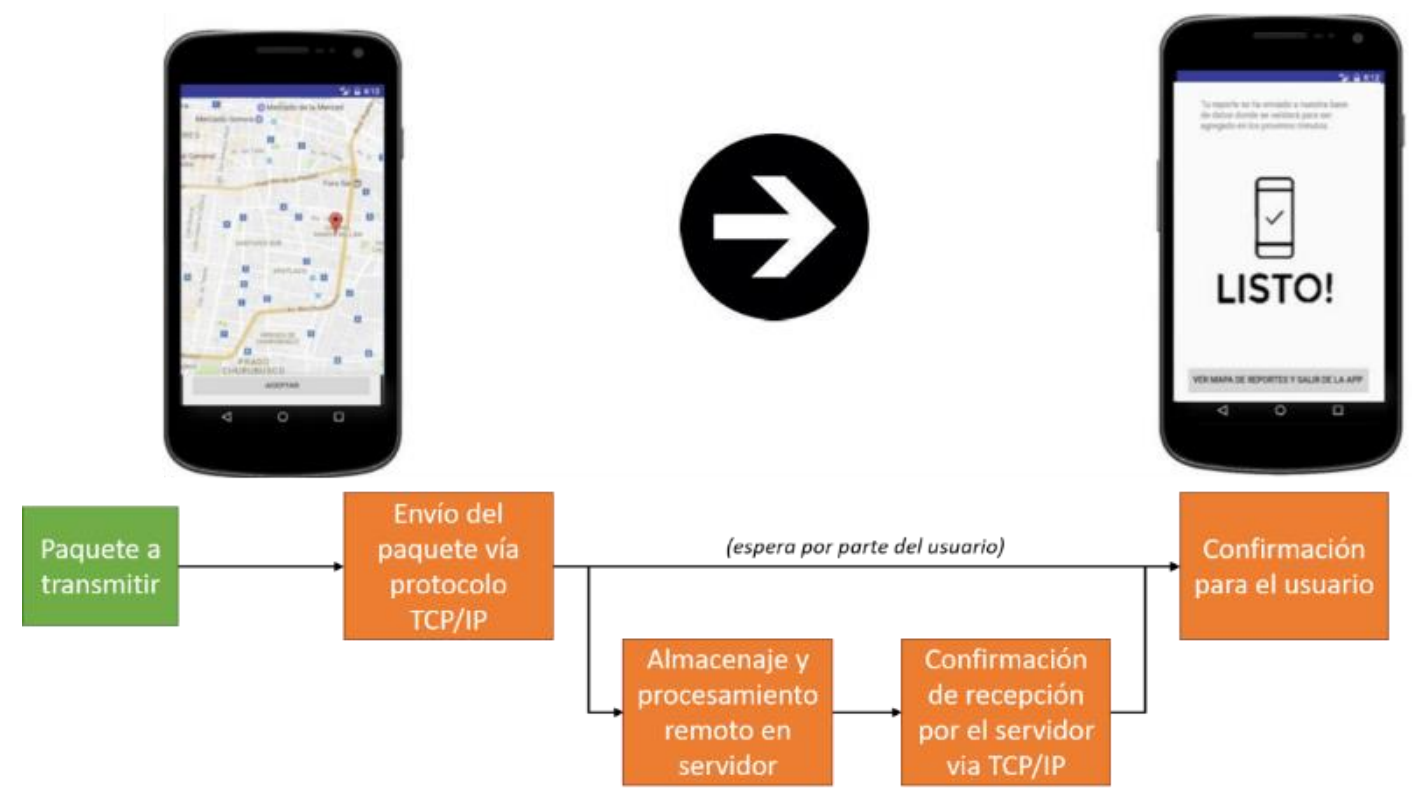

Fig. 3. Algoritmo de transmisión de la información recopilada en el dispositivo del usuario.

\section{METODOLOGÍA: TRATAMIENTO DE LA INFORMACIÓN.}

Con la finalidad de alcanzar el objetivo de analizar los patrones de la actividad criminal y de proveer de información al usuario final, es requerida una estrategia de tratamiento de la información recopilada, la cual se ha dividido en este artículo en siete fases. La primera etapa consiste en la recopilación de datos por parte del usuario que ha sido víctima o testigo de un delito, el cual consiste en obtener tanto los detalles del incidente delictivo, como los datos del usuario para identificar la procedencia del reporte y evitar reportes apócrifos. Algunos de los detalles necesarios para el reporte son la posición geográfica y temporal (latitud, longitud, fecha y hora), el modus operandi (asalto en automóvil, con arma blanca, con arma de fuego, homicidio, etc...), y una descripción del incidente. Detalles de esta fase se muestran en la sección anterior. 
Una vez obtenidos los datos, la segunda fase requiere realizar una validación de los mismos. Este trabajo se enfoca en las demarcaciones de la delegación Iztacalco en la Ciudad de México, por lo que el incidente solo será georrefenciado si el marcador del reporte y la posición del dispositivo que lo capturan coinciden con dicha zona geográfica. Esto es con la finalidad de evitar que un usuario intente levantar un reporte apócrifo con información que no coincide con su posición actual. Futuras implementaciones pueden enfocarse en distintos filtros como es el histórico de reportes del usuario, la calidad de la información de descripción, o la discrepancia entre otros reportes en el mismo lugar y hora.

Como tercera fase, se requiere la transformación de los datos filtrados en un archivo con el formato estándar CSV, el cual será alojado dentro de un servidor de almacenamiento y procesamiento, con la finalidad de clasificar los delitos reportados dentro de un SIG. Con base en dicha fase, la cuarta etapa de la estrategia se basa en la generación de tablas, estadísticas y puntos de control, con el objetivo de indizar la latitud y longitud a un mapa acorde a la información obtenida. Esta información permitirá no solamente el obtener el plano cartográfico, sino también registros temporales de como el crimen se mueve y se erradica por la ciudad.

Una vez indizada la información obtenida de los usuarios, la quinta etapa transforma los datos al cartograma mencionado. El cartograma con los datos añadidos podrá ser descargado de forma gráfica en el SIG, visualizándose como puntos de eventos. Cada punto en el mapa vinculará a los respectivos detalles que describen cada incidente en específico. La sexta etapa consiste en la generación de modelos de proximidad entre eventos, así como la delimitación de zonas por colonias cuadrantes de la policía de la ciudad de México. Otra de las funcionalidades de dicha etapa consiste en mostrar filtros por clasificación de delitos y con ellos crear consultas para visualizar por tipo de incidente, área geográfica, o temporalidad.

Finalmente, la séptima etapa consiste en la transmisión de tanto los mapas como los modelos de proximidad y datos a los usuarios, de tal manera que la aplicación -de nombre GeoSecurityMX- permita a los usuarios el observar tanto los incidentes reportados, como el evitar las zonas donde los delitos se cometen con frecuencia. De esta manera también las autoridades serán capaces de tener información en tiempo real que les permita reducir la tasa de crímenes en la ciudad. Un resumen de la metodología mencionada puede observarse en la figura 4.

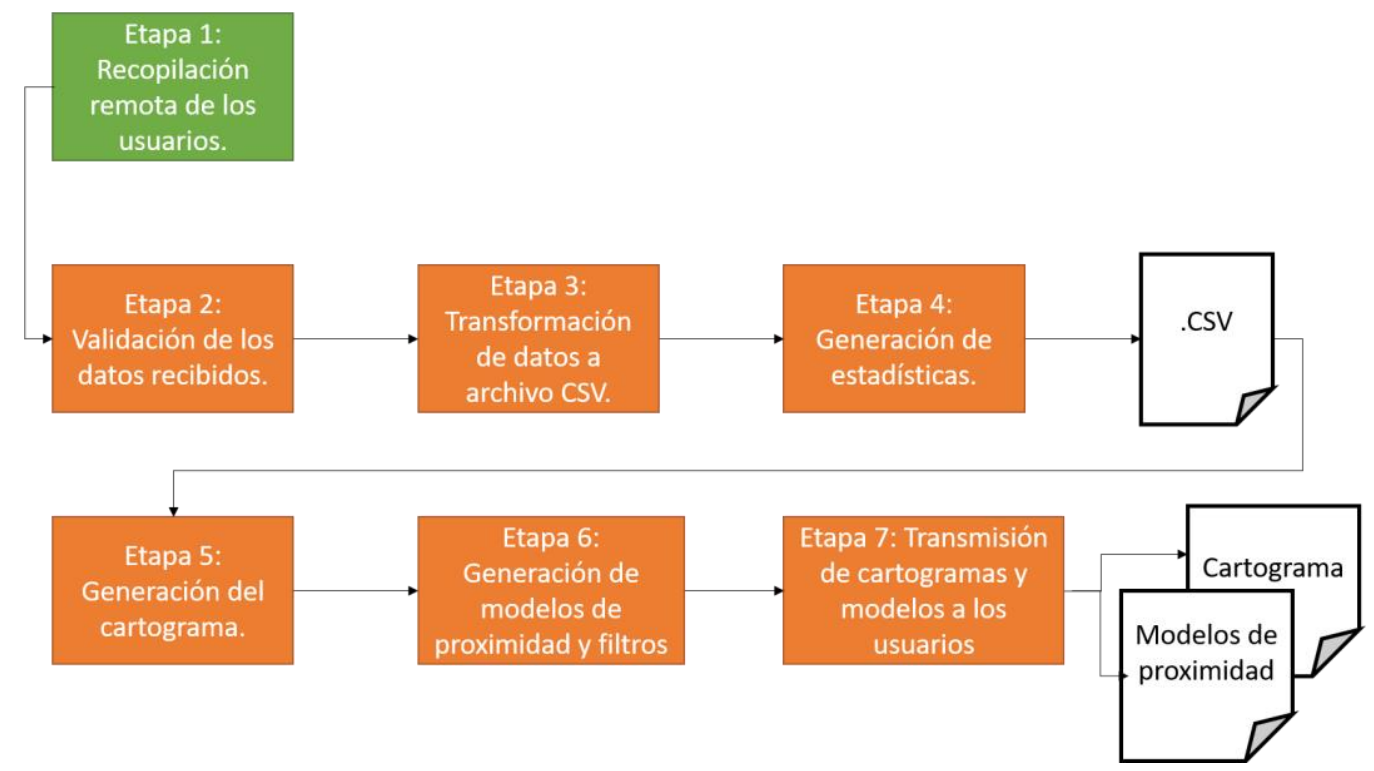

Fig. 4. Metodología de tratamiento de la información. La fase 1 se lleva a cabo en los dispositivos de los usuarios, mientras que la fase 2 a 7 se lleva a cabo dentro de un servidor remoto.

\section{RESULTADOS Y DISCUSION}

Se generaron diferentes reportes geo-referenciados dentro de la delegación Iztacalco para probar la eficacia de la metodología propuesta, en la cual se obtuvieron como resultados distintos mapas cartográficos, observables tanto para los usuarios de la aplicación GeoSecurityMX como para los analistas del comportamiento de crimen. Un ejemplo de los mapas de delitos generados dentro de la interfaz del sistema puede observarse en la figura 5.

En cada mapa de delitos generado, se cuentan con diversas capas superpuestas que permiten obtener información sobre el comportamiento del crimen. Algunas de estas capas muestran las demarcaciones de las delegaciones, los delitos cometidos, los cuadrantes policiacos y las colonias de la delegación Iztacalco (mismas que son sub-áreas contenidas dentro de la delegación). Dichas capas pueden ser observadas en 
superposición, para determinar la intersección entre ellas y obtener información relevante, como la presencia (o ausencia) de elementos policiacos, en contraposición con la actividad delictiva.
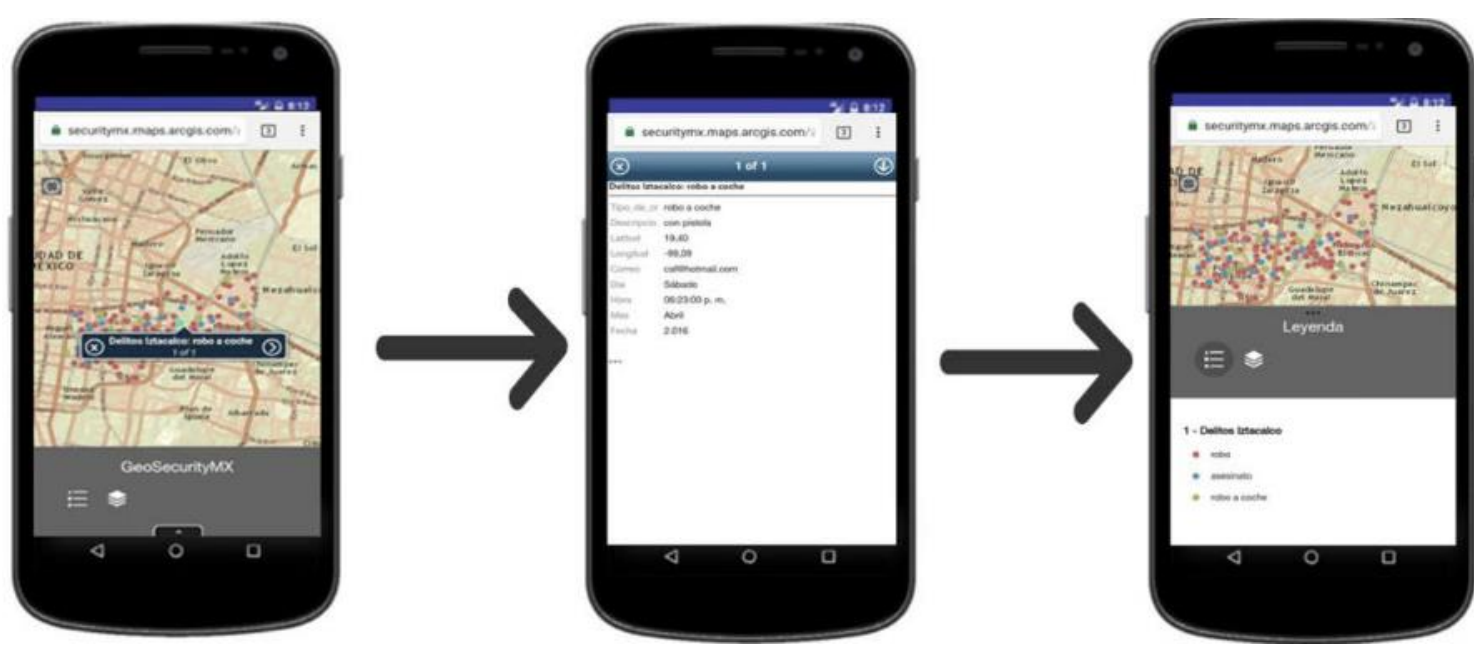

Fig. 5: Mapa de delitos observables en el sistema GeoSecurityMX.

Cada uno de los puntos obtenidos dentro del mapa de delitos observables es capaz de desplegar una descripción breve del incidente causado, el cual es recopilado por la metodología mencionada en las secciones previas. De forma puntual, es posible observar la fecha, hora, tipo de delito, descripción, y -de forma opcional, dependiendo de la implementación- el usuario que reporta el robo.

La información obtenida tanto de los usuarios como de las demarcaciones propias de la división política de la Ciudad de México tiene diversas formas de presentarse al usuario final. Los delitos y reportes son mostrados como puntos en un mapa, y la división política es mostrado mediante un conjunto de polígonos superpuestos. La manera de realizar el trazado de ubicación de cada punto se basa en el sistema de coordenadas WGS 1984 (GEOINT, 1984). Sin embargo, el resultado más importante que genera el sistema propuesto es la construcción de representaciones simplificadas del delito, que facilitan el análisis del movimiento criminal para ser utilizados tanto por la fuerza policial como para los usuarios de la aplicación.

En la figura 6 se despliega, a manera de ejemplo, una vista general de los reportes delictivos de la delegación Iztacalco. Se toma como base el mapa cartográfico, y con dicha base se despliegan los reportes en puntos específicos de forma simultánea. El sondeo mostrado en dicha figura muestra que los delitos tuvieron diversos modus operandi, por ende, se muestra la simbología en el mapa correspondiente a cada modus operandi. La segunda fase de la metodología propuesta consiste en el filtrado de reportes. Para efectos de este estudio, se considera que un reporte debe ser filtrado si se encuentra fuera de los límites de la delegación Iztacalco. Si bien es cierto que el filtrado de reportes y la validación puede depender de las políticas de cada implementación, la figura 7 muestra a manera de ejemplo los lugares donde se genera el reporte -en otras palabras, la ubicación real del dispositivo que hace el reporte-.

El objetivo de este filtrado es discernir si las ubicaciones reportadas coinciden con las ubicaciones reales con cierto grado de aproximación. Esto permite evitar que un delito reportado dentro de la demarcación tenga un reporte en otra zona geográfica (por ejemplo, otro país). Esta implementación puede considerar un cierto umbral de distancia de semiverseno -entre dos latitudes y longitudes- para validar que el reporte corresponde a un caso real. Un tercer resultado consiste en el cómputo de los modelos de proximidad: Un delito puede causar una influencia radial variable, dependiendo del delito que se ha consumado. Dicha influencia puede variar dependiendo de la implementación; para el caso de este modelo, se han implementado dos influencias radiales de 100 y 200 metros para cada uno de los casos. En la figura 8 se pueden observar casos de homicidio en color rojo, asaltos en color verde y asaltos a vehículo en color amarillo. La razón principal de generar los mapas con zonas de influencia es crear un conjunto de áreas que sean potencialmente riesgosas para los usuarios del sistema, al igual que se generen zonas donde la focalización de las autoridades policiales pueda ser requerida.

Cabe destacar que existen dos mecanismos para construir las zonas de influencia. Uno es por medio de la distancia euclideana en un plano bidimensional y otro método es la construcción por medio de la distancia geodésica, tomando en cuenta la curvatura terrestre (Olaya, 2014). Esta distancia también es conocida como la distancia de semiverseno, y ambas maneras de calcular la influencia radial pueden ser construidas dentro del sistema propuesto. 


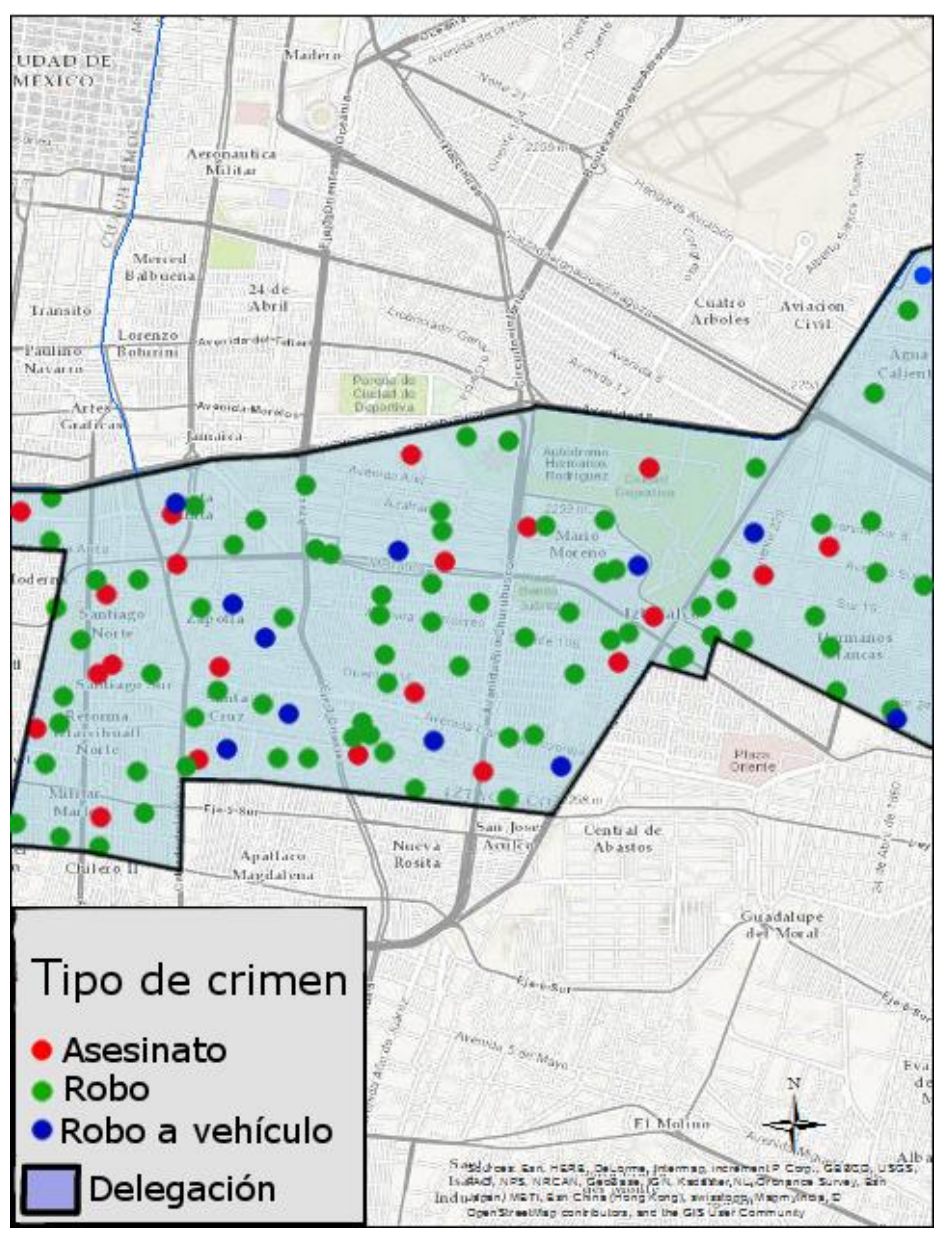

Fig. 6: Mapa de análisis para los delitos de la delegación Iztacalco. Se muestran diferentes tipos de crimen cometidos con su respectiva señalética.

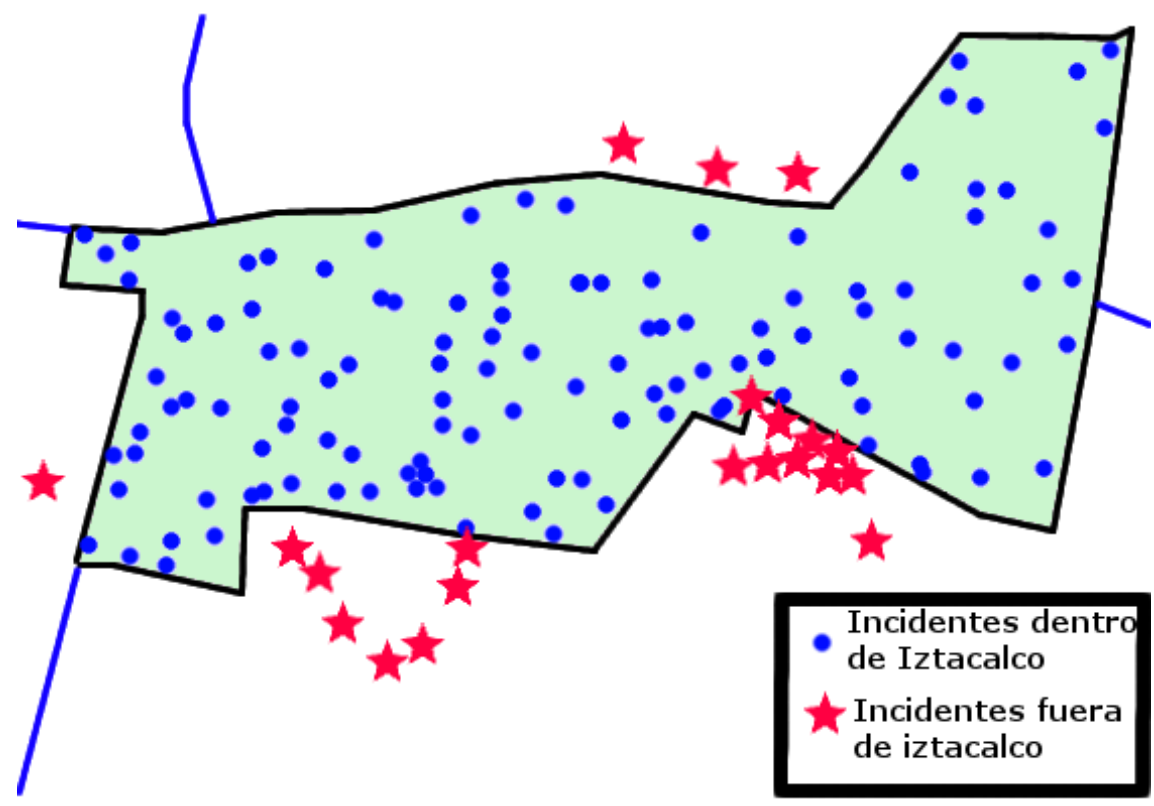

Fig. 7: Mapa de análisis para los delitos de la delegación Iztacalco: Delitos reportados fuera de la delegación, en yuxtaposición con los delitos dentro de la delegación.

\section{DISCUSION FINAL}

Los Sistemas de Información Geográfica (SIG) son herramientas relativamente nuevas que han demostrado proveer un gran soporte a tareas de inteligencia geográfica como es el caso de manejo de tráfico, localización 
geográfica y planeación urbana. El presente trabajo tiene como finalidad combinar tres elementos tecnológicos con los sistemas de información geográfica: Los dispositivos móviles, el enfoque de extracción de información por parte de los usuarios -también llamado crowdsourcing-, y el procesamiento de latitudes y longitudes dentro de un SIG.

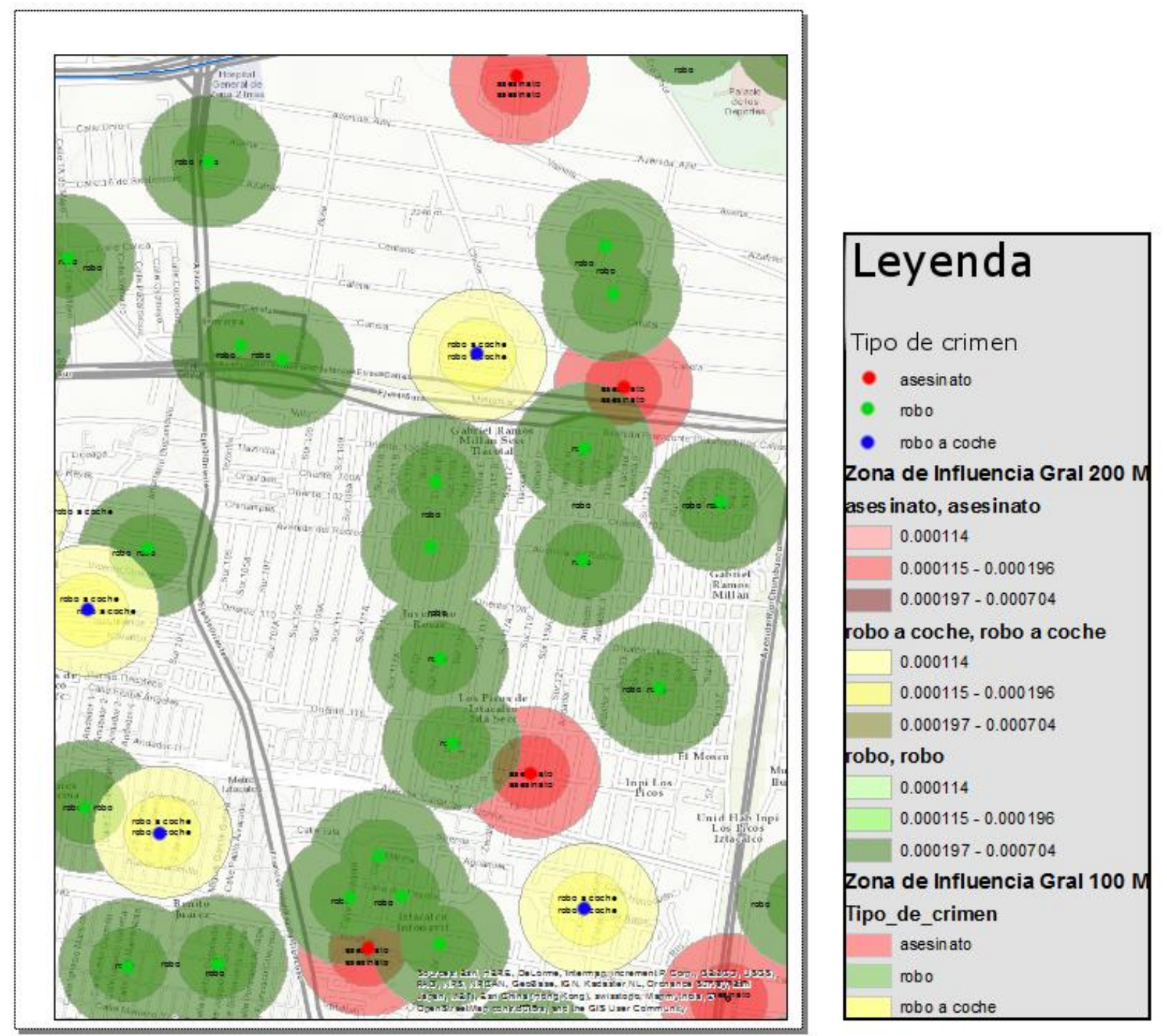

Fig. 8: Mapa de análisis generado: Zonas de influencia a 100 y 200 metros. El centro es un incidente, mientras que la periferia es una zona que podría no tener adecuado control policial.

El presente trabajo muestra una metodología de implementación que permite combinar los elementos antes mencionados para proveer a los mismos usuarios tanto de cartogramas como de modelos de proximidad. Esta información tiene la finalidad de prevenir a los usuarios de un percance delictivo cercano a su periferia, al igual que puede ser proveído a las fuerzas policiacas y a las autoridades para focalizar esfuerzos en ciertas zonas para reducir el crimen. Los resultados obtenidos constan de mapas cartográficos que están constituidos por distintas capas superpuestas, las cuales pueden servir para realizar un análisis rápido y útil tanto para usuarios como para autoridades. Un ejemplo de superposición de capas que puede arrojar nuevas acciones es la acción delictiva versus las zonas de patrullaje policial y de acción de la autoridad.

Si bien es cierto que la delegación Iztacalco es un espacio geográfico acotado para este problema, no hay impedimento alguno para realizar la implementación en distintas delegaciones, estados, o inclusive países. La flexibilidad en el filtrado de la información generada puede permitir el bloqueo de reportes apócrifos, la comparativa entre dos o más reportes, e inclusive el bloqueo de diferentes usuarios que constantemente reportan información falsa o de dudosa calidad. Futuros desarrollos pueden incluir, además de mejoras en la metodología de la información generada, algoritmos de inteligencia artificial que permitan predecir el comportamiento delictivo con base en los históricos que este sistema arroja con el paso del tiempo. Esto con la finalidad de dificultar la perpetración de un crimen y mejorar los servicios de seguridad de las zonas urbanas que implementen el sistema propuesto. 


\section{CONCLUSIONES}

Con base en la discusión final, es posible enumerar las conclusiones del presente de la siguiente manera. 1) Es posible y viable la combinación de las tecnologías móviles, la infraestructura desarrollada en las zonas urbanas en materia de telecomunicaciones y el modelo de crowdsourcing para afrontar los problemas de delincuencia en la Ciudad de México; 2) Los resultados obtenidos muestran planos cartográficos que permiten no solamente el asistir a los mismos usuarios de la aplicación móvil para prevenir el delito y sus zonas de influencia, sino también para advertir a las autoridades de los puntos en donde los delitos se cometen recurrentemente y 3) la flexibilidad de la implementación permite no solamente la creación de planos cartográficos y puntos donde la delincuencia se focaliza, sino también permite implementar diversos filtros que ayuden a la validación de la información y a la reducción de reportes apócrifos.

\section{REFERENCIAS}

Alvarado, A., Organized Crime in Latin American Cities. The Case of Mexico City, Revista Latinoamericana de Estudios de Seguridad, 19(1), 129-145 (2016)

Bailey, J., Crimen e impunidad: Las trampas de la seguridad en México. 1aㅡ., Editorial Debate, México (2014)

Brabham, D.C., Crowdsourcing as a Model for Problem Solving, an introduction and cases, Convergence: International Journal of Research into New Media Technologies, 14(1), 75-90 (2008)

Boba, R., Introductory guide to Crime Analysis and Mapping, Police foundation, Crime mapping laboratory. U.S. Department of Justice (2001)

Cruz G. L., Structure of perception in public safety crisis, Universidad Nacional Autónoma de México y Liberabit, Revista Peruana de Psicología, 18(1), 37-44 (2012)

Duansheng, C., D. Yongquan y Z. Yibao, Design and Implementation of a Location Awareness System for Field Police Work" International Conference on Geoinformatics, 1-4 (2009)

Ekins, S., A.J. Williams, Reaching out to Collaborators: Crowdsourcing for Pharmaceutical Research, Pharmaceutical Research, 27(3), 393-395 (2010)

Estellés-Arolas, E., Towards an integrated crowdsourcing definition, Journal of Information Science, 38(2), 189-200 (2012)

Fan, W. y C. Ye, Design and study on police intelligence analysis system based on GIS, International Conference on Computational Intelligence and Software Engineering, (1) 1-4 (2009)

Gao, H., G. Barbier y R. Goolsby, Harnessing the Crowdsourcing power of social media for disaster relief, IEEE Inteligent systems, 26(3), 10-14 (2011)

GEOINT Sciences Office, World Geodetic System 1984 (1984)

Khasraghi, H. J. y M.J. Tarokh, Efficient Business Process Reengineering with Crowdsourcing, International Journal of Applied Information Systems, 2(7), 1-5 (2012)

Olaya, V., Sistemas de Información Geográfica (2014)

Ruiz M. A., La transición a la Inseguridad. Entrevista con John Bailey, Este País, 291(1), $23-26$ (2015)

Vilalta, C.J., Fear of crime in public transport: Research in Mexico City, Crime prevention and community safety, 13(3), 171-186 (2011)

Yan X., X. Li y D. Lin, Assessing Urban Public Security Based on Remote Sensing and GIS", International Conference on Geoinformatics (23), 1-6 (2015)

Zhong, B., L. Niu y H. Chen, Design and implement of $3 \mathrm{G}$ mobile police system", International Conference on Consumer Electronics, Communicacions and Networks. (2), 1752-1755 (2012) 
